\title{
Absence of Kondo resonance in high-resolution photoemission spectra of monocrystalline $\mathrm{Fe}_{1-x} \mathrm{Co}_{x} \mathrm{Si}$
}

\author{
D. Zur,* D. Menzel, I. Jursic, and J. Schoenes \\ Institut für Physik der Kondensierten Materie, Technische Universität Braunschweig, Mendelssohnstrasse 3, \\ D-38106 Braunschweig, Germany \\ L. Patthey \\ Paul Scherrer Institut, CH-5232 Villigen PSI, Switzerland \\ M. Neef, K. Doll, and G. Zwicknagl \\ Institut für Mathematische Physik, Technische Universität Braunschweig, Mendelssohnstrasse 3, D-38106 Braunschweig, Germany
}

(Received 21 February 2007; published 6 April 2007)

\begin{abstract}
$\mathrm{Fe}_{1-x} \mathrm{Co}_{x} \mathrm{Si}$ single crystals with different Co concentrations were grown using the tri-arc Czochralski technique. Samples with 0,5 , and 20 at. $\%$ of Co were studied with photon energies of 21.2 and $40.8 \mathrm{eV}$ with spectral resolutions as high as $8 \mathrm{meV}$ at the Fermi level. For FeSi, a full gap of $28 \mathrm{meV}$ has been found, which closes with $5 \%$ of Co. In the range between 20 and $150 \mathrm{eV}$, energy dependent angle-resolved measurements in the [111] direction were made for FeSi. Through a comparison with theoretical calculations, the states at the Fermi energy can be directly assigned to Fe $3 d$ states. No sign of Kondo resonance is found.
\end{abstract}

DOI: 10.1103/PhysRevB.75.165103 PACS number(s): 71.20.Be, 73.20.At, 75.20.Hr, 79.60.Bm

\section{INTRODUCTION}

The unusual properties of the narrow-gap semiconductor FeSi continue to intrigue many theoreticians and experimentalists. This material has an insulating and nonmagnetic ground state with an increasing susceptibility when raising the temperature. ${ }^{1}$ Above $500 \mathrm{~K}$, the magnetic susceptibility shows a Curie-like behavior. Although band-structure calculations produce a gap of the correct order of magnitude, ${ }^{2}$ problems in describing the very peculiar susceptibility behavior with the computed density of states exist. ${ }^{1-3}$ Various proposals have been made to overcome these problems, ranging from spin-fluctuation models ${ }^{4-6}$ to strongly correlated models. ${ }^{7}$ The latter were inspired by the observation that the density of states required to fit the susceptibility is very similar ${ }^{3}$ to the one which can result from $f$ - $d$ hybridization in Kondo insulators. ${ }^{8}$ Other models using LDA $+U$ (local density approximation with Coulomb correlation $U$ ) calculations stress the fact that FeSi is a small-gap semiconductor close to a first-order transition to a ferromagnetic metal. ${ }^{9}$ A low-energy metastable metallic phase is predicted by density-functional calculations, which account for nonlocal exchange. ${ }^{10}$

This proximity to a magnetic ground state led Anisimov et $a l .{ }^{11}$ to study the effect of substituting Ge for Si. While it is difficult to grow $\mathrm{FeSi}_{1-x} \mathrm{Ge}_{x}$ single crystals due to a peritectic segregation of the constituents, substitutions of Fe with some other $3 d$ metals are quite straightforward. Interestingly, the substitution of $\mathrm{Co}$ for $\mathrm{Fe}$ also leads to dramatic changes of the electronic and magnetic properties. Thus, $\mathrm{Fe}_{1-x} \mathrm{Co}_{x} \mathrm{Si}$ is paramagnetic for $x<0.05$ and turns into a ferromagnetic metal by doping with more than 5 at. $\%$ Co. ${ }^{12,13}$ This magnetic transition is accompanied by the closing of the band gap. For $x>0.8, \mathrm{Fe}_{1-x} \mathrm{Co}_{x} \mathrm{Si}$ becomes paramagnetic again, whereas $\mathrm{CoSi}$ is a diamagnetic semimetal. ${ }^{14}$

To date, the experimental results are also not conclusive. From various thermodynamic and spectroscopic studies, gap energies ranging from 0 to approximately $100 \mathrm{meV}$ have been derived. Concentrating here on the generally very powerful optical and photoelectron spectroscopies, one notes the following: Optical spectroscopy investigations ${ }^{13,15-17}$ agree reasonably well on the gap size $E_{g}$ of $50-60 \mathrm{meV}$, but they disagree on the redistribution of the oscillator strength when the gap opens. ${ }^{13,15}$ Whether this intensity accumulates within about $4 E_{g}$ or only over a much larger energy scale has been seen as a key issue to disentangle the bandlike models from the Kondo lattice models.

Photoemission is the most direct way to study the density of states near the Fermi energy $\left(E_{F}\right)$. Unfortunately, the presently available data are contradictory. Earlier measurements were limited by energy resolution and did not show a gap ${ }^{18}$ or only a very small gap. ${ }^{19,20}$ Later, Breuer et al. ${ }^{21}$ found a pseudogap of $75 \mathrm{meV}$. However, this gap is not fully developed and the existence of a full band gap which is larger than $1 \mathrm{meV}$ is excluded. The absence of a full gap is attributed to surface states which remain metallic at low temperature or alternatively to residual impurities acting as Anderson localized states at $E_{F}$. Most recently, Ishizaka et $a{ }^{22}$ reported laser photoemission results which, due to the low excitation energy of about $7 \mathrm{eV}$, are expected to be less surface sensitive than the data of Breuer et al. taken at $\sim 20$ and $40 \mathrm{eV} .^{21}$ Although the probing depth is estimated to be $\sim 100 \AA$ for these measurements and Ishizaka et al. observe a deeper gap of 50-60 meV, the density of states still does not reach zero in the gap, which the authors attribute to the existence of impurities.

To resolve the experimental discrepancies and to shed light on the origin of the gap, we have performed highresolution, low-temperature photoemission experiments with various excitation energies of a synchrotron on high quality $\mathrm{Fe}_{1-x} \mathrm{Co}_{x} \mathrm{Si}$ single crystals with $x=0,0.05$, and 0.20.

We find a fully developed gap of approximately $28 \mathrm{meV}$ for $x=0$ with definitely zero density of states at $E_{F}$ and just 


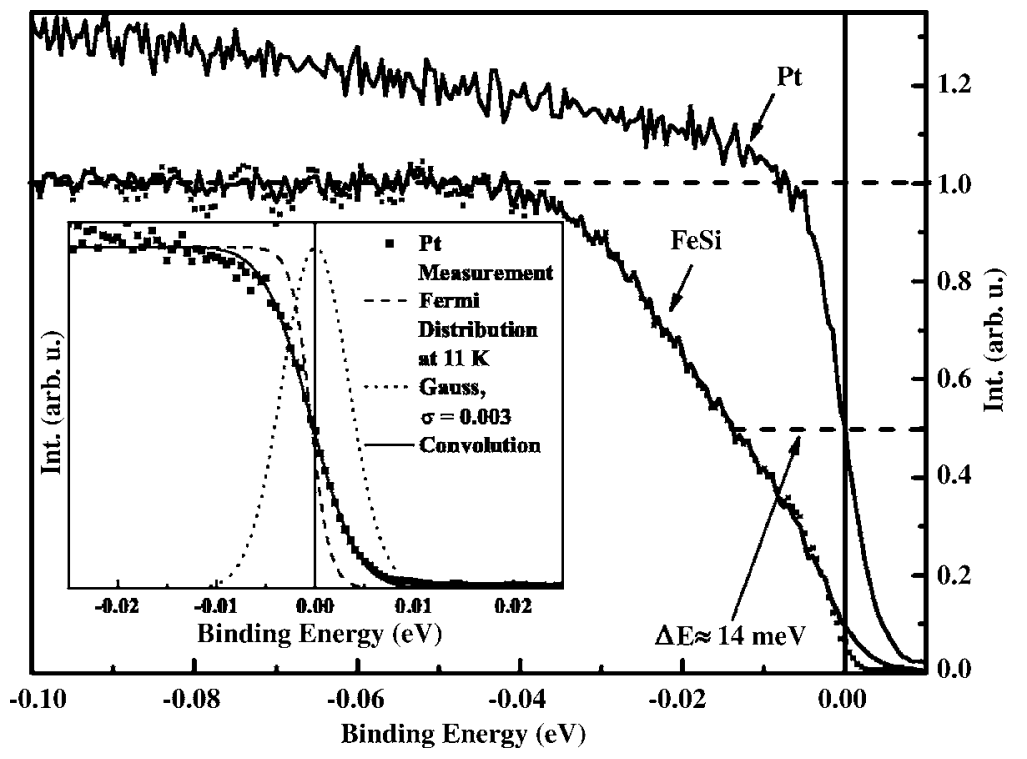

FIG. 1. A comparison between the FeSi (line, measurement; dots, deconvolution) and the $\mathrm{Pt}$ measurements shows the absence of states at the Fermi energy for FeSi. Inset: The Pt spectrum is well-fitted with a convolution of a Fermi distribution at $11 \mathrm{~K}$ and a Gauss peak with $\sigma=0.003$. an exiguous number of impurities. This gap decreases to zero for $x=0.05$. For the highest cobalt concentration studied $(x$ $=0.20)$, the density of states at $E_{F}$ is clearly metallic. A density-functional calculation indicates that the states near $E_{F}$ are predominantly of Fe $d$ character. The fact that the experimentally observed dispersion is of the order of $1 \mathrm{eV}$ is incompatible with the Kondo picture. The latter predicts strongly renormalized bands ${ }^{23}$ whose widths of $\sim 50-70 \mathrm{meV}$ (Ref. 3) reflect the characteristic energy scale $k_{B} T^{*}$ related to the anomalous variation with temperature of the susceptibility.

\section{EXPERIMENT}

The high-resolution photoemission experiments were carried out at the Swiss Light Source of the Paul Scherrer Institute. The photoelectron spectrometer SCIENTA SES-2002 provided an energy resolution of approximately $8 \mathrm{meV}$. In order to define exactly the position of the Fermi energy, a Pt reference sample was used as a free-electron-like material. During the measurements, the temperature of the samples was kept at $11 \mathrm{~K}$ to prevent the very sharp electronic structure to be washed out due to thermal broadening. The instrumental broadening of the spectrometer was derived from the shape of the Fermi function of the Pt reference and was used to deconvolute the FeSi spectra. The $\mathrm{Fe}_{1-x} \mathrm{Co}_{x} \mathrm{Si}$ single crystals $(x=0,0.05$, and 0.2$)$ were grown by the tri-arc Czochralski method. From susceptibility measurements at low temperature we derived for $\mathrm{FeSi}$ an impurity concentration of $0.17 \%$, which is lower than the values reported before. ${ }^{15,24,25}$ The surface of the crystals were cleaned by heating to $700{ }^{\circ} \mathrm{C}$ in the UHV chamber. We refrained from sputtering the surface in order to keep a good crystallinity at the surface $^{21}$ and to prevent a contamination with Ta, which was used to mount the crystals onto the sample holder. A lowenergy electron-diffraction (LEED) analysis confirmed the cleanness and the crystallinity of the surface. Additionally, we found a clearly resolved $\mathrm{Si} 2 p$ doublet in the x-ray photoemission spectroscopy spectrum, which also demonstrates that the surface was clean without any indication of oxidation. ${ }^{26}$ The cleanness check was accomplished for all Co concentrations.

\section{RESULTS AND DISCUSSION}

Figure 1 shows a spectrum taken on a [111] oriented FeSi crystal near the gap for an incident photon energy of $21.2 \mathrm{eV}$. The comparison with the associated Pt spectrum shows a clear absence of states at the Fermi level for FeSi. Previous measurements on FeSi showed some electronic states with a reduced intensity at the Fermi energy, which were attributed to a disordered and, therefore, metallic surface superimposed to a full-band-gap bulk contribution. ${ }^{21}$ Another reason for the metallic behavior could be the contribution of impurities in bulk FeSi which act as Anderson localized states, leading to a nonzero density of states at the Fermi energy. ${ }^{27}$ In our spectrum, these "metallic" states are adumbrated in the diminutive shoulder at $10 \mathrm{meV}$. This indicates either a better crystallinity of our surface, which is supported by the LEED observation, and/or single crystals containing less impurities.

The broadening of the spectra is caused by a convolution with an apparatus function, which is approximated with a Gaussian function. A fit of the Pt spectrum (inset in Fig. 1) gives a convolution of the Fermi edge at $11 \mathrm{~K}$ with a Gaussian peak with $\sigma=0.003 \mathrm{eV}$. The FeSi spectrum is deconvoluted (dots in Fig. 1) by the van Cittert method ${ }^{28}$ (20 iterations) with this Gaussian peak. Evidently, there are no states observable at the Fermi energy. ${ }^{21,22}$ This corroborates the high quality of our sample as was already derived from the low residual susceptibility. Assuming an intrinsic semiconductor, a fully opened gap of $\sim 28 \mathrm{meV}$ is found, taking the full width at half maximum. ${ }^{29}$ This value constitutes rather a lower limit considering the fact that previous experiments at higher temperatures have shown an asymmetric gap with $E_{F}$ closer to the top of the valence band than the bottom of the conduction band. ${ }^{22}$ The gap closes with $5 \%$ Co (Fig. 2). This concentration is known to be at the borderline between semi- 


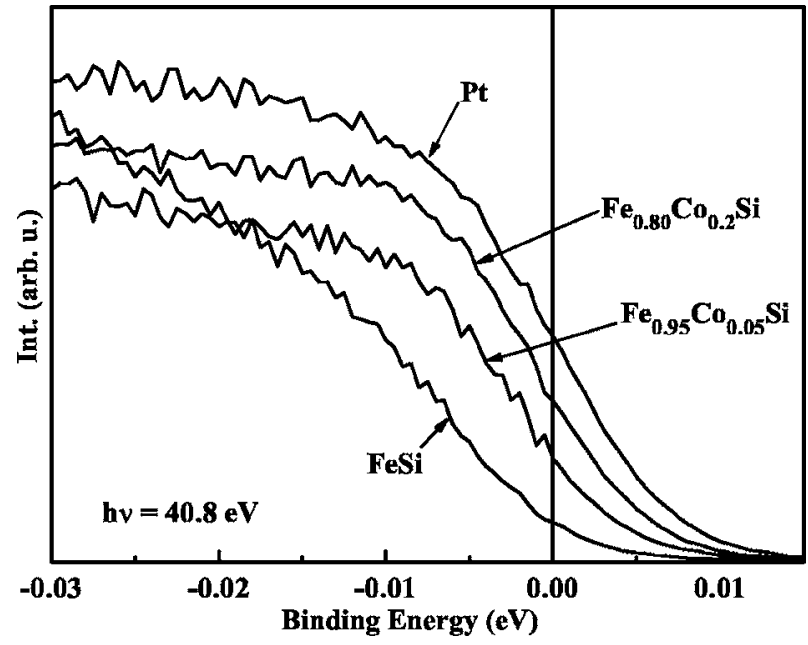

FIG. 2. The states of $\mathrm{Fe}_{1-x} \mathrm{Co}_{x} \mathrm{Si}$ at the Fermi energy are filled up with increasing Co concentration. The spectra have been normalized to the intensity at the binding energy of $-150 \mathrm{meV}$.

conducting and ferromagnetic metallic behaviors. For $20 \%$ $\mathrm{Co}$, the density of states is clearly metallic and the sample orders ferromagnetically at $T_{C}=40 \mathrm{~K} \cdot{ }^{30}$

Using photon energies between 20 and $150 \mathrm{eV}$, energy dependent measurements along the [111] direction toward the $R$ point with an integration angle of $1^{\circ}$ around the normal emission were performed on a (111) FeSi surface (Fig. 3). While the peak at about $-2 \mathrm{eV}$ (feature 2) in Fig. 3 does not move with increasing photon energy, the peak near the Fermi edge (feature 1) shifts to lower binding energy. This indicates that in the region of the $\Gamma$ point (low photon energies), states are close to the Fermi energy and shift to higher absolute values of binding energies near the $R$ point (higher photon energies). Comparing this result with the theory (Fig. 4), one can relate features 1 and 2 in both figures and assign them to Fe $3 d$ states. Furthermore, the photoionization cross section

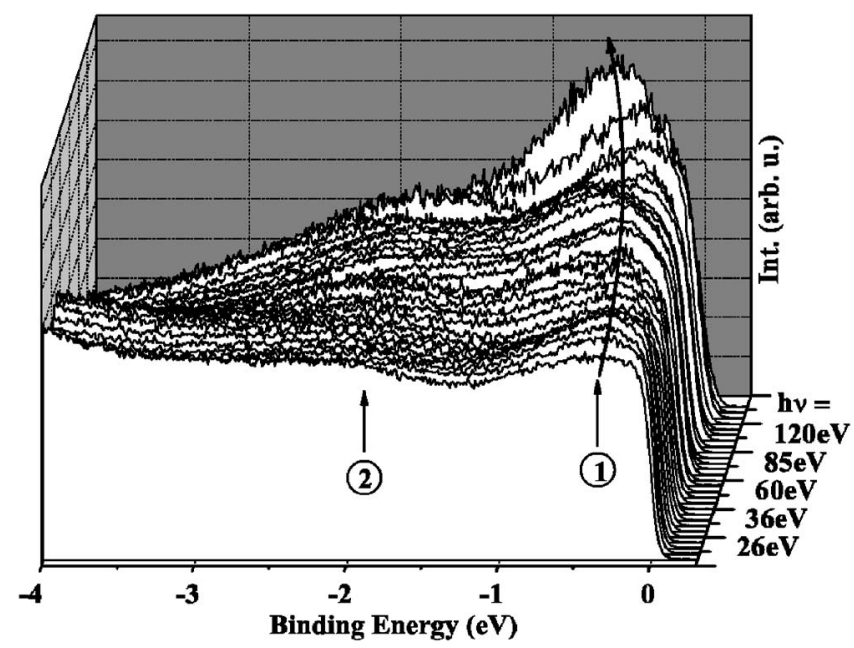

FIG. 3. Energy dependent spectra in the photon energy range of $20-150 \mathrm{eV}$ taken with a spectral resolution of $70 \mathrm{meV}$. The numbered features correspond to the energy bands in the computed FeSi band structure (Fig. 4). The big arrow is a guide for the eyes, illustrating the energy shift of peak 1 .
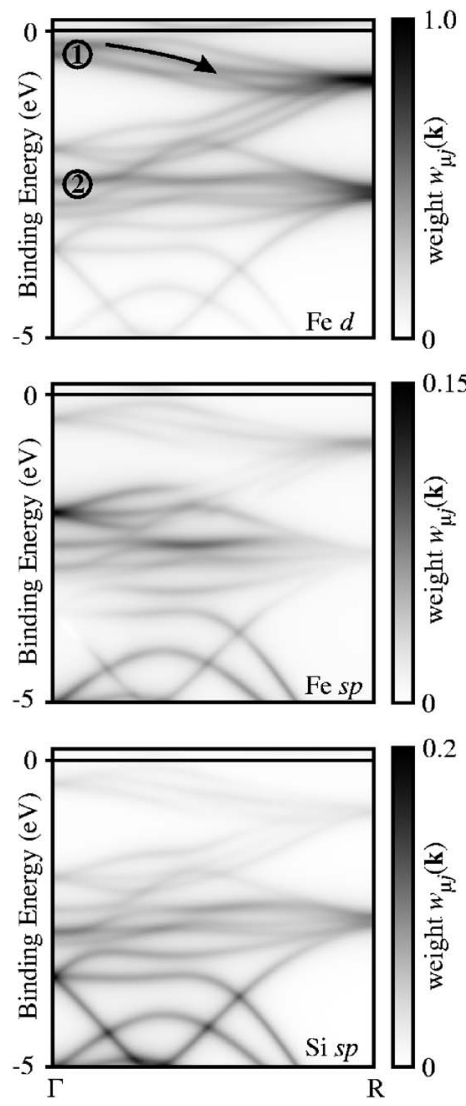

FIG. 4. Computed FeSi band structure along $\Gamma-R$. The bands were broadened using a Lorentzian, and the weights were obtained by projecting the bands on the Fe $d$ (top), Fe $s p$ (middle), and Si $s p$ (bottom) basis functions. Note the different gray scales in the three plots. The arrow illustrates the energy shift of peak 1 in Fig. 3.

for Fe $3 d$ states is larger at higher energies than for $\mathrm{Si} 3 p$ or $3 s$ states, which is mirrored in the measurement by the increasing intensity of feature 1 with increasing photon energies. ${ }^{31}$

\section{THEORY}

Density-functional calculations with the gradientcorrected functional of Perdew and Wang were performed using the code CRYSTAL2003. ${ }^{32}$ The basis functions are Gaussian-type orbitals centered at the position of the atoms, as in Ref. 10.

FeSi is considered in its experimentally established B20 structure with a lattice parameter $a=4.493 \AA$ and the internal coordinates $u_{\mathrm{Fe}}=0.1358$ and $u_{\mathrm{Si}}=0.844$.

The density-functional band structure is well established and is in good agreement with the experiment, describing $\mathrm{FeSi}$ as a narrow-gap insulator. ${ }^{2}$ For a more detailed comparison with the experiment, the weight $w_{\mu j}(\mathbf{k})$ of the basis function $\mu$ at the reciprocal-lattice point $\mathbf{k}$ of band number $j$ given by ${ }^{32}$

$$
w_{\mu j}(\mathbf{k})=\sum_{\nu} \sum_{\mathbf{g}} S_{\mu \nu}(\mathbf{k}) a_{\mu j}^{*}(\mathbf{k}) a_{\nu j}(\mathbf{k}) \exp (i \mathbf{k g})
$$

was calculated. Here, $S_{\mu \nu}$ is the overlap matrix of the basis functions indicated by $\mu$ and $\nu, \mathbf{g}$ are the direct lattice vec- 
tors, and the set of eigenvectors $a_{\mu i}$ are the solutions of the Kohn-Sham equations. These bands were then broadened around the eigenvalues $\epsilon_{j}(\mathbf{k})$, using a Lorentzian $\frac{1}{\pi} \frac{\eta w_{\mu j}}{\left[\omega-\epsilon_{j}(\mathbf{k})\right]^{2}+\eta^{2}}$ with a broadening $\eta=0.1 \mathrm{eV}$. The results for the [111] direction are displayed in Fig. 4.

\section{CONCLUSION}

The absence of any sharp structure near $E_{F}$ and the good agreement between experiment and theory lead us to conclude that the one-particle band-structure calculation is a better starting point for the description than the Kondo model, in spite of the appeal of the latter. Only Park et al. ${ }^{18}$ who did not observe a gap at all, have ever reported a sharp peak of $0.2 \mathrm{eV}$ width near $E_{F}$. This peak may be related to a very flat band in the particular direction probed in their experiment which, considering the narrow band 1 along the $\Gamma-R$ direction, does not seem unlikely. Of course, correlations have to be included to account for several of the peculiar properties of $\mathrm{FeSi}$, such as the rapid closing of the gap with temperature and the anomalous temperature dependence of the susceptibility. On the basis of our present data, we cannot decide whether spin fluctuations which have received support from polarized-neutron experiments ${ }^{6}$ or the addition of the local Coulomb repulsion ${ }^{9}$ will be the better approach. Further experimental and theoretical work is clearly needed to solve the many puzzles of this intriguing material.

\section{ACKNOWLEDGMENTS}

This work was performed at the Swiss Light Source, Paul Scherrer Institut, Villigen, Switzerland. We are grateful to the machine and beamline groups whose outstanding efforts have made these experiments possible. This research project has been supported by the European Commission under the 6th Framework Programme through the Key Action: Strengthening the European Research Area, Research Infrastructures, Contract No. RII3-CT-2004-506008.
*Electronic address: d.zur@tu-braunschweig.de

${ }^{1}$ V. Jaccarino, G. K. Wertheim, J. H. Wernick, L. R. Walker, and S. Arajs, Phys. Rev. 160, 476 (1967).

${ }^{2}$ L. F. Mattheiss and D. R. Hamann, Phys. Rev. B 47, 13114 (1993).

${ }^{3}$ D. Mandrus, J. L. Sarrao, A. Migliori, J. D. Thompson, and Z. Fisk, Phys. Rev. B 51, 4763 (1995).

${ }^{4}$ Y. Takahashi and T. Moriya, J. Phys. Soc. Jpn. 46, 1451 (1979).

${ }^{5}$ S. N. Evangelou and D. M. Edwards, J. Phys. C 16, 2121 (1983).

${ }^{6}$ K. Tajima, Y. Endoh, J. E. Fischer, and G. Shirane, Phys. Rev. B 38, 6954 (1988).

${ }^{7}$ Castor Fu and S. Doniach, Phys. Rev. B 51, 17439 (1995).

${ }^{8}$ P. Wachter and G. Travaglini, J. Magn. Magn. Mater. 47\&48, 423 (1985).

${ }^{9}$ V. I. Anisimov, S. Y. Ezhov, I. S. Elfimov, I. V. Solovyev, and T. M. Rice, Phys. Rev. Lett. 76, 1735 (1996).

${ }^{10}$ M. Neef, K. Doll, and G. Zwicknagl, J. Phys.: Condens. Matter 18, 7437 (2006).

${ }^{11}$ V. I. Anisimov, R. Hlubina, M. A. Korotin, V. V. Mazurenko, T. M. Rice, A. O. Shorikov, and M. Sigrist, Phys. Rev. Lett. 89, 257203 (2002).

${ }^{12}$ N. Manyala, Y. Sidis, J. F. DiTusa, G. Aeppli, D. P. Young, and Z. Fisk, Nature (London) 404, 581 (2000).

${ }^{13}$ M. A. Chernikov, L. Degiorgi, E. Felder, S. Paschen, A. D. Bianchi, H. R. Ott, J. L. Sarrao, Z. Fisk, and D. Mandrus, Phys. Rev. B 56, 1366 (1997).

${ }^{14}$ J. H. Wernick, G. K. Wertheim, and R. C. Sherwood, Mater. Res. Bull. 7, 1431 (1972).

${ }^{15}$ Z. Schlesinger, Z. Fisk, Hai-Tao Zhang, M. B. Maple, J. F. DiTusa, and G. Aeppli, Phys. Rev. Lett. 71, 1748 (1993).

${ }^{16}$ H. Ohta, S. Kimura, E. Kulatav, S. V. Halilov, T. Nanba, M. Motokawa, M. Sato, and K. Nagasaka, J. Phys. Soc. Jpn. 63, 4206 (1994).

${ }^{17}$ A. Damascelli, K. Schulte, D. van der Marel, and A. A. Menovsky, Phys. Rev. B 55, R4863 (1997).
${ }^{18}$ C.-H. Park, Z.-X. Shen, A. G. Loeser, D. S. Dessau, D. G. Mandrus, A. Migliori, J. Sarrao, and Z. Fisk, Phys. Rev. B 52, R16981 (1995).

${ }^{19}$ A. Chainani, T. Yokoya, T. Morimoto, T. Takahashi, S. Yoshii, and M. Kasaya, Phys. Rev. B 50, 8915 (1994).

${ }^{20}$ T. Saitoh, A. Sekiyam, T. Mizokama, A. Fujimori, K. Ito, H. Nakamura, and M. Shiga, Solid State Commun. 95, 307 (1995).

${ }^{21}$ K. Breuer, S. Messerli, D. Purdie, M. Garnier, M. Hengsberger, Y. Baer, and M. Mihalik, Phys. Rev. B 56, R7061 (1997).

${ }^{22}$ K. Ishizaka, T. Kiss, T. Shimojima, T. Yokoya, T. Togashi, S. Watanabe, C. Q. Zhang, C. T. Chen, Y. Onose, Y. Tokura, and S. Shin, Phys. Rev. B 72, 233202 (2005).

${ }^{23}$ G. Zwicknagl, Adv. Phys. 41, 203 (1992).

${ }^{24}$ E. Arushanov, M. Respaud, J. M. Broto, J. Leotin, S. Askenazy, Ch. Kloc, E. Bucher, and K. Lisunov, Phys. Rev. B 55, 8056 (1997).

${ }^{25}$ S. Paschen, E. Felder, M. A. Chernikov, L. Degiorgi, H. Schwer, H. R. Ott, D. P. Young, J. L. Sarrao, and Z. Fisk, Phys. Rev. B 56, 12916 (1997).

${ }^{26}$ S. Dreiner, M. Schürmann, C. Westphal, and H. Zacharias, Phys. Rev. Lett. 86, 4068 (2001).

${ }^{27}$ M. B. Hunt, M. A. Chernikov, E. Felder, H. R. Ott, Z. Fisk, and P. Canfield, Phys. Rev. B 50, 14933 (1994).

${ }^{28}$ P. H. van Cittert, Z. Phys. 69, 298 (1931).

${ }^{29}$ In Ref. 21 the gap is taken at the energy, where the spectrum deviates from the straight line. With this definition, our full gap amounts to $75 \mathrm{meV}$, in full agreement with Ref. 21.

${ }^{30}$ D. Menzel, D. Zur, and J. Schoenes, J. Magn. Magn. Mater. 272276, 130 (2004).

${ }^{31}$ J. J. Yeh and I. Lindau, At. Data Nucl. Data Tables 32, 1 (1985).

${ }^{32}$ V. R. Saunders, R. Dovesi, C. Roetti, R. Orlando, C. M. ZicovichWilson, N. M. Harrison, K. Doll, B. Civalleri, I. J. Bush, Ph. D'Arco, and M. Llunell, CRYSTAL 2003 User's Manual (University of Torino, Torino, 2003). 